\title{
Finite sets of data compatible with multidimensional inequality measures
}

\author{
José C. R. Alcantud \\ Facultad de Economía y Empresa, Universidad de Salamanca, E 37008 \\ Salamanca, Spain
}

\begin{abstract}
By using a general solution to the problem of extending a preorder conditional on a list of ex-ante comparisons between pairs, we ellucidate when a finite set of predetermined comparisons can be incorporated to a multidimensional inequality measure even if the population size is variable.
\end{abstract}

Key words: Multidimensional inequality, preorder, ordering, ordering extension, conditional extension.

Email address: jcr@usal.es (José C. R. Alcantud ).

URL: http://web.usal.es/jcr

Tel. +34-923-294640.

Fax. +34-923-294686. (José C. R. Alcantud ). 


\section{Introduction.}

The need for measures of multidimensional inequality arises when more than one criterion has to be applied in order to evaluate for example, welfare attached to different populations, household income inequality, or goodness for energy populations. A large number of analytical tools have been designed to compare distributions on this basis. Several proposals address the question from the viewpoint of attaching numerical values to each distribution (cf., e.g., Savaglio [20] or Weymark [29] for general surveys). The most frequently used relative inequality index is the Gini index, a flexible tool for analysis that is also used e.g., as node splitting measure for decision tree construction (cf., Chandra and Varghese [8] or Koprinska et al. [13] for recent references) or to derive welfare functions that can be used to compute energy welfare (cf., Ok et al. [18, Sect. 4.3]). But univariate inequality indices like the Gini index, the Theil index [24], or Atkinson's [6] indices do not give a full picture of the extent of inequality between groups of agents. This leads to constructions of multi-attribute inequality indices like the index developed by Maasoumi in [15-17]. It is constructed in two stages. In each step choices are made based on information theory: General Entropy measures are selected for both stages. Likewise, multi-attribute versions of e.g., Atkinson's index have been proposed in the literature (cf., Tsui [25]). Other methods for comparing pairs of distributions include dominance principles whose main handicap is incompleteness (cf., e.g., Atkinson and Bourguignon [7] and Savaglio [19]).

The fact that a method of comparison in terms of inequality performs admittedly well is not incompatible with some degree of slackness in its prescriptions. For example: the U.S. Census Bureau historical table for measures of household income inequality in the country [26] claims that in the period 1988 to 1991, it increased according to the Gini measure but decreased according to the Theil measure. The same mixed evidence is observed in the period 1998 to 1999. The set of data that produced the historical table is the same. Nonetheless, if a researcher wants to use them to support the ex-ante prescription that household income inequality increased in the 1988-91 and 1998-99 lapses she can do it by means of a measure with good properties (namely, the Gini index), and similarly she can decide to support the opposite position (by appealing to the Theil index). In fact she can also use the same data to support the view that inequality increased in the 1988-91 period but decreased in the 1998-99 period by appealing to an orthodox procedure like Atkinson's measure with parameter 0.75.

Obviously, this slackness can not be extended arbitrarily without violating desirable postulates. We are not aware of any analysis of the degree of slackness that is allowed when normative properties are imposed on complete methods of comparison. Our contribution intends to put forward this problem and present a first solution by referring to the recent approach by Savaglio [19], which has the remarkable feature that the assumption of fixed population size is dropped. Thus, in our proposal we first discuss properties that are desirable for a criteria in the current context. Then we consider a finite list of comparisons between distributions of goods or attributes to different populations (of possibly different sizes). Such list captures a given assessment of the inequality that those distributions convey to their respective populations. Finally, we 
check if this prescription is compatible with the existence of a criterion with the selected properties. In order to do so we take advantage of the recent Alcantud [2] (v. Demuynck [9] for a broader discussion), where a general problem relating to extensions of preorders has been provided.

This paper is organized as follows. Section 2 gives some basic notation. Then in Section 3 we brief the reader on the conditional extension problem and its solution (Subsection 3.1), explain some specialized notation (Subsection 3.2), present some normative postulates (Subsection 3.3), describe the model (Subsection 3.4), and check for independence of its axiomatics (Subsection 3.5). Subsection 3.6 contains the solution to our problem directly from the arguments and results of Subsection 3.1. We summarize and address some related topics in Section 4.

\section{Basic notation.}

Let $\mathbf{X}$ be a non-empty set. A binary relation $R$ on $\mathbf{X}$ is a subset of $\mathbf{X} \times \mathbf{X}$. As is standard, $x R y$ is shorthand for $(x, y) \in R$. A reflexive and transitive relation is called a preorder, also called quasiordering. An ordering is a complete preorder.

The asymmetric factor $P_{R}$ and the symmetric factor $I_{R}$ of $R$ are defined by

$$
\begin{aligned}
& P_{R}=\{(x, y) \in X \times X \mid x R y \text { and not } y R x\} \\
& I_{R}=\{(x, y) \in X \times X \mid x R y \text { and } y R x\}
\end{aligned}
$$

If $R$ is a complete preorder then $I_{R}$ is an equivalence relation. The shorthands $P$ for $P_{R}, \widetilde{P}$ for $P_{\widetilde{R}}, \hat{P}$ for $P_{\hat{R}}, \ldots$ or $I$ for $I_{R}, \widetilde{I}$ for $I_{\widetilde{R}}, \hat{I}$ for $I_{\hat{R}}, \ldots$ are common use.

If $R$ and $S$ are binary relations on $\mathbf{X}$ and $R \subseteq S$ then we say that $R$ is contained or included in $S$. An extension of $R$ binary relation on $\mathbf{X}$ is a binary relation $S$ on $\mathbf{X}$ such that $R \subseteq S$ and $P_{R} \subseteq P_{S}$. Szpilrajn's Theorem ([23], also [4, Th. 1.7]) assures that every preorder can be extended to a complete preorder, i.e., it has an ordering extension.

\section{An axiomatic approach to multidimensional inequality with initial con- straints.}

In this Section we introduce the model that we intend to analyze and then solve our main question by means of recent advances in the theory of ordering extensions. Therefore we first brief the reader on the relevant approach to this technical problem in Subsection 3.1. Then we proceed to state and discuss the axioms under inspection and to set our model. After checking for independence of the postuates we prove the main result of the paper. 


\subsection{A fundamental result on conditional ordering extensions.}

Extending preorders to complete preorders has much appeal because it permits to work with relations that incorporate enough information as to apply significant tools of analysis, such as e.g., maximality results or utility assignments. Szpilrajn's theorem and its generalizations and variants are very often quoted and applied in many branches of mathematics and social sciences (for a very detailed description we refer to Andrikopoulos [5]). However, to the purpose of empirical contrast of the model we typically perform tests when only a finite amount of information is gathered. For that reason the applied researcher needs to be able to check when a finite number of exogenous comparisons can be matched with one such extension. The exact conditions under which we can extend a given preorder to a complete preorder conditional on a finite list of predetermined comparisons were given in Alcantud [2]. The following concept is the key to specify such solution.

Definition 1 Let $X_{I}=\left(a_{1}, \ldots, a_{n}, b_{1}, \ldots, b_{n}\right)$ be an ordered list of possibly repeated elements of $\mathbf{X}$, and $R$ a preorder on $\mathbf{X}$. The $R^{A}$ relation associated with $X_{I}$ and $R$ is given by $a_{i} R^{A} a_{j}$ if and only if $a_{i} R b_{j}$.

Remark 1 It is worth stating some particular cases of Definition 1. Under its assumptions, $R^{A}$ is irreflexive if and only if $a_{i} R b_{i}$ is false for each $i=1, \ldots, n$, because $a_{i} R b_{i}$ amounts to $a_{i} R^{A} a_{i}$. If $n=1$ then $R^{A}$ is acyclic if and only if $a_{1} R b_{1}$ is false. And if $n=2$ then $R^{A}$ is acyclic if and only if the assertions $a_{1} R b_{1}, a_{2} R b_{2}$, and $\left(a_{1} R b_{2}\right.$ plus $a_{2} R b_{1}$ ) are all false.

The solution to that particular extension of Szpilrajn's theorem can be stated in the following terms.

Theorem 1 Let $R$ be a preorder on a set $\mathbf{X}$. Let $X_{I}=\left(a_{1}, \ldots, a_{n}, b_{1}, \ldots, b_{n}\right)$ be an ordered list of possibly repeated elements of $X$. The following statements are equivalent:

a) There is $\widetilde{R}$ ordering extension of $R$ such that $b_{i} \widetilde{P} a_{i}$ for each $i=1, \ldots, n$, where $\widetilde{P}$ denotes the asymmetric part of $\widetilde{R}$.

b) $R^{A}$ associated with $\left(a_{1}, \ldots, a_{n}, b_{1}, \ldots, b_{n}\right)$ and $R$ is acyclic.

Besides, implication $a) \Rightarrow b$ ) requires that $\widetilde{R}$ contains $R$ only.

Theorem 1 transforms the original question into a discrete problem, that is, define a simple binary relation on a finite set (informally: the collection of elements on which some conditioning is imposed, named $X_{I}$ ) and check if it has cycles. Example 1 below illustrates this technique (for applications and further examples see Alcantud [2]).

Example 1 For any fixed set $\mathbf{U}$ we let $R$ be the preorder on the set of subsets of $\mathbf{U}$ given by inclusion. Suppose $\mathbf{U}=\{0,1,2,3\}$. Let $A_{1}=\{0,1,2\}, B_{1}=\{0,1\}, A_{2}=\{1\}$, and $B_{2}=\{0,2,3\}$.

(a) Can we produce $\tilde{R}$ complete preorder on $\mathbf{U}$ that extends $R$ and satisfies $B_{1} \tilde{P} A_{1}$ 
and $B_{2} \tilde{P} A_{2}$ simultaneously?

According to Theorem 1 and Remark 1, we must examine if $A_{1} \subseteq B_{1}, A_{2} \subseteq B_{2}$, and $\left(A_{1} \subseteq B_{2}\right.$ plus $\left.A_{2} \subseteq B_{1}\right)$ are all false. Because this is the case we conclude that the answer is in the affirmative.

As a matter of fact, $A \tilde{R} B$ if and only if $\operatorname{card}(A) \leqslant \operatorname{card}(B)$ solves question (a).

(b) Let $\overline{B_{2}}=\{0,1,2,3\}$. Can we produce $\tilde{R}$ complete preorder on the set of subsets of $\mathbf{U}$ that extends $R$ and satisfies $B_{1} \tilde{P} A_{1}$ and $\overline{B_{2}} \tilde{P} A_{2}$ simultaneously?

Now the statement $A_{1} \subseteq \overline{B_{2}}$ plus $A_{2} \subseteq B_{1}$ is true. Therefore, no such construction can be obtained.

\subsection{Further notation and definitions.}

Henceforth all matrices have real values. The rank of a matrix $A$ is represented by $\operatorname{rank}(A)$. A permutation matrix is a matrix such that each row and column has exactly one non-null cell and its value is 1 . Premultiplying (resp., postmultiplying) a matrix by a permutation matrix results into a permutation of its rows (resp., its columns). A matrix $P=\left(p_{i j}\right)_{i j} \in M_{p \times m}(\mathbb{R})$ is row-stochastic if $p_{i j} \geqslant 0$ for each possible $i, j$ and $p_{i 1}+\ldots .+p_{i m}=1$ for each $i=1, \ldots, p$. Any permutation matrix is row-stochastic, and any row-stochastic matrix is a fuzzy matrix (Lur et al. [14, Sect. 1]). The inverse of a permutation matrix is its transpose, therefore another permutation matrix. The product of row-stochastic matrices is row-stochastic.

$M_{m \times n}(\mathbb{R})$ represents the set of all $m \times n$ matrices with real values. Henceforth $n$ is fixed and captures the commodities or goods or attributes that each individual in the community can possess or enjoy: we label them $1, \ldots, n . M_{n}$ represents the set of all matrices with $n$ columns and real values. For expository convenience we adopt the economic terminology, thus each $A=\left(a_{i j}\right)_{i, j} \in M_{m \times n}(\mathbb{R}) \subseteq M_{n}$ is interpreted as an allocation of goods to a population of $m$ individuals of the society, which assigns $a_{i j}$ units of the $j$ 'th good to agent $i$ of that population. We are concerned with orderings on $M_{n}$ that meet both rational and external prerequisites with regard to the amount of inequality that the allocations convey.

\subsection{Axioms.}

Let $R$ be a preorder on $M_{n}$. In our framework we are interested in the following list of rationality restrictions for $R$ that are in line with its interpretation as "it is at most as sparse as". Their implications are examined thereafter.

Axiom $A$ (Attribute-independence). If $T$ is a permutation matrix of size $n \times n$ then $A I_{R}(A T)$ for each $A \in M_{n}$.

Axiom $P$ (Preference for average allocations). For each $A \in M_{p \times n}(\mathbb{R})$ and $Q \in$ 
$M_{m \times p}(\mathbb{R})$ row-stochatic: $(Q A) R A$.

Axiom $C$ (Compatibility with the rank). For each $A, B \in M_{n}$ : if $A R B$ then $\operatorname{rank}(A) \leqslant \operatorname{rank}(B)$.

Axiom $S$ (Scale Invariance). For each $A \in M_{n}, A I_{R}(k A)$ when $k>0$.

Attribute-independence means that the level of disparity in the distribution that a list of attributes produces does not vary if we shuffle the attributes. For example: for a single agent having $(1,5)$ is similar to having $(5,1)$ from the strict point of view of how spread out is the distribution of goods he is assigned. This postulate is admittedly controversial in some contexts (e.g., if the first attribute is "health" and the second is "income") but important cases speak for its plausibility (e.g., when all personal attributes are perfect substitutes). Although we do not intend to endorse it, since we aim at producing a possibility result the more properties we can guarantee the more relevant it is.

Preference for average allocations says that assigning averages of a list of individual endowments is at most as spread out as assigning those initial endowments to a collective of individuals, irrespective of how many individuals are endowed with averages. It implies the following usual assumption that the name of the agents should not affect the level of inequality.

Axiom $A N$ (Anonymity). If $T$ is a permutation matrix of size $m \times m$ then $A I_{R}(T A)$ for each $A \in M_{m \times n}(\mathbb{R}) \subseteq M_{n}$.

Compatibility with the rank is in the spirit of Property C in Savaglio [19]. It means that a comparison on the basis of disparity can not declare $A$ at most as unequally distributed as $B$ when $B$ has fewer linearly independent rows, since rows incorporate each individual's assignment within the population to which commodities are allocated.

Scale invariance means that changing the unit of measure should not alter the inequality assessment. If the attributes are monetary endowments, this means that the choice of the currency should not affect the comparisons.

Remark 2 It is trivial that if $R$ is a preorder that verifies Axiom $A$ (resp., AN, $P$, or $S$ ) then whenever $R \subseteq R^{\prime}$ the relation $R^{\prime}$ verifies it too.

\subsection{The model.}

A consistent with inequality ordering-also CWI ordering- on the set of all matrices with $n$ columns is a complete preorder on that set that agrees with Axioms $A, A N, P$, $C$, and $S^{1}$. For example, the rank provides one such CWI ordering: define $r$ on $M_{n}$ by $A r B$ if and only if $\operatorname{rank}(A) \leqslant \operatorname{rank}(B)$.

1 Although $A N$ is redundant in this context we find it instructive to list it along the properties due to its undeniable appeal. 
In this work we are concerned with the more restrictive problem of finding complete preorders on $M_{n}$ that agree with Axioms $A, A N, P, C$, and $S$ and satisfy a list of initial constraints. We call them conditional CWI orderings and of course, $r$ is not a conditional CWI ordering with regard to any possible list of initial constraints.

Remark 3 Savaglio [19] studied a particular majorization concept for matrices representing a group of agents endowed with amounts of a fixed number of commodities. It underlies a procedure for constructing inequality indices associated with sublinear functions, and is called vp-majorization. Such proposal consists of declaring $A \ll B$ if and only if there is $P$ row-stochastic with $A=P B$, whenever $A \in M_{p \times n}(\mathbb{R}), B \in M_{m \times n}(\mathbb{R})$. This is a preorder, i.e., a reflexive and transitive (but incomplete) relation.

Let us confront this position with the axioms above. For one thing, « agrees with Axioms $A N, C$ and $P$ (which implies reflexivity by itself). For another, this incomplete relation verifies neither Axiom $S$ (quite trivially) nor Axiom $A$, thus it is not CWI. To prove it, just observe that if we let $T_{0}=\left(\begin{array}{ll}0 & 1 \\ 1 & 0\end{array}\right)$ and $A_{0}=\left(\begin{array}{ll}2 & 1 \\ 4 & 2\end{array}\right)$ then the matrices $A_{0}$ and $A_{0} T_{0}=\left(\begin{array}{ll}1 & 2 \\ 2 & 4\end{array}\right)$ are unrelated by $\ll$.

In order to solve the issue we have raised we need a preliminary definition.

Definition 2 For any $A, B$ matrices with $n$ columns, let $A \lesssim B$ mean that there are $k>0$, some $Q$ row-stochastic matrix and some $T$ permutation matrix with $A=k Q B T$.

It is clear that $\lesssim$ is a preorder on $M_{n}$ that contains $\ll$ and agrees with Axioms $A$, $A N, P, C$, and $S$. It is not complete because if we let

$$
F=\left(\begin{array}{ll}
1 & 1 \\
1 & 1
\end{array}\right) \text { and } G=\left(\begin{array}{ll}
2 & 1 \\
2 & 1
\end{array}\right)
$$

then neither $F \lesssim G$ nor $G \lesssim F$ is true. All the binary relations that contain $\lesssim$ satisfy Axioms $A, A N, P$ and $S$ by virtue of Remark 2 .

Remark 4 Although $\ll \subseteq r, r$ is not an extension of $\ll$. The reason is that for

$$
A^{\prime}=\left(\begin{array}{rr}
1 & 0 \\
1.5 & 0.5
\end{array}\right), B^{\prime}=\left(\begin{array}{ll}
1 & 0 \\
2 & 1
\end{array}\right)
$$

we have $A^{\prime} I_{r} B^{\prime}$ but nonetheless $A^{\prime} P_{\ll} B^{\prime}$, that is, $A^{\prime} \ll B^{\prime}$ holds and $B^{\prime} \ll A^{\prime}$ does not. Indeed, the fact $\left(\begin{array}{cc}1 & 0 \\ 0.5 & 0.5\end{array}\right) B^{\prime}=A^{\prime}$ proves $A^{\prime} \ll B^{\prime}$. The assertion $Q^{\prime} A^{\prime}=B^{\prime}$ 
entails $Q^{\prime}=\left(\begin{array}{rr}1 & 0 \\ -1 & 2\end{array}\right)$, which is not row-stochastic thus $B^{\prime} \ll A^{\prime}$ does not hold.

The same example shows that despite $\lesssim \subseteq r,{ }^{2} r$ is not an extension of $\lesssim$. Now the fact $A^{\prime} \lesssim B^{\prime}$ derives from $A^{\prime} \ll B^{\prime}$, and assuming $B^{\prime} \lesssim A^{\prime}$ produces a contradiction as follows. We claim the existence of $k>0, Q$ row-stochastic matrix and $T$ permutation matrix with $A^{\prime}=k Q B^{\prime} T$. Two cases arise:

$$
\text { either } T \text { is the identity matrix or } T=\left(\begin{array}{ll}
0 & 1 \\
1 & 0
\end{array}\right)
$$

In words: either postmultiplying $B^{\prime}$ by $T$ results into $B^{\prime}$ or into a permutation of its columns. Both cases yield trivial contradictions therefore $A^{\prime} P_{\lesssim} B^{\prime}$ in spite of $A^{\prime} I_{r} B^{\prime}$.

Suppose that a social planner assumes that the inequality that assignments convey must be modelled by means of a CWI ordering. In principle he may want to assume that $F$ conveys strictly less inequality than $G$ without violating that assumption, not for normative reasons but e.g., because he feels bound to a political programme or by agreement with other parties. That produces an initial constraint. Can he reconcile all these requirements? In order to fully assess our model it is relevant to check that Axioms $A, P, C$, and $S$ are independent, which we proceed to prove by producing suitable examples in Subsection 3.5. Then in Subsection 3.6 we provide an answer to the question when the number of constraints is arbitrary.

\subsection{Independence of the axioms.}

Example 2 Axioms $P, C$, and $S$ together do not imply Axiom $A$.

We just need to adapt the construction in Remark 3 as follows. Let $A \ll^{\prime} B$ if and only if there are $k>0$ and some $P$ row-stochastic with $A=k P B$, whenever $A \in M_{p \times n}(\mathbb{R}), B \in M_{m \times n}(\mathbb{R})$. Because $\ll \subseteq \ll^{\prime}$ and $\ll$ verifies Axiom $P$ then Remark 2 assures that $\ll^{\prime}$ must verify it too. It is clear that $\ll^{\prime}$ verifies Axioms $C$ and $S$. Nonetheless the argument in Remark 3 shows that $\ll^{\prime}$ does not verify Axiom $A$.

Example 3 Axioms $A, P$, and $C$ together do not imply Axiom $S$.

We just need to adapt the construction in Definition 2 as follows. For any $A, B$ matrices with $n$ columns, let $A \lesssim B$ mean that there are some $Q$ row-stochastic matrix and some $T$ permutation matrix with $A=Q B T$. Now Axiom $S$ fails to hold true but Axioms $A, P$, and $C$ are verified easily.

2 We use the fact that the rank of a product of matrices is lesser than or equal to the minimum of the ranks of the factors, by Sylvester's rank inequality (v., Horn and Johnson, [11, Sect. 0.4.5]). 
Example 4 Axioms $A, C$, and $S$ together do not imply Axiom $P$.

Let $A \preccurlyeq B$ if and only if there are $k>0$ and some $T, T^{\prime}$ row-stochastic with $A=$ $k T B T^{\prime}$, whenever $A \in M_{p \times n}(\mathbb{R}), B \in M_{m \times n}(\mathbb{R})$. It is clear that $\preccurlyeq$ verifies Axioms $A$, $C$ and $S$. Nonetheless $\preccurlyeq$ does not verify Axiom $P$ because if we let

$$
A=\left(\begin{array}{ll}
1 & 1 \\
0 & 0
\end{array}\right) \text { and } Q=\left(\begin{array}{cc}
\frac{1}{2} & \frac{1}{2} \\
\frac{1}{2} & \frac{1}{2}
\end{array}\right)
$$

then $Q A=Q=\left(\begin{array}{cc}\frac{1}{2} & \frac{1}{2} \\ \frac{1}{2} & \frac{1}{2}\end{array}\right) \preccurlyeq A$ is impossible.

Example 5 Axioms $A, P$, and $S$ together do not imply Axiom $C$.

We modify the construction in Definition 2 as follows. There exist $M_{1}, M_{2}$ matrices with 2 columns, such that $\operatorname{rank}\left(M_{1}\right)<\operatorname{rank}\left(M_{2}\right)$ and both $M_{1} \lesssim M_{2}$ and $M_{2} \lesssim M_{1}$ are false. For example,

$$
M_{1}=\left(\begin{array}{ll}
1 & 1
\end{array}\right) \text { and } M_{2}=\left(\begin{array}{rr}
-1 & -1 \\
0 & -1
\end{array}\right)
$$

For each $A, B \in M_{2}$, let $A \lesssim " B$ mean either $A \lesssim B$ or $\left(A \lesssim M_{2}\right.$ and $\left.M_{1} \lesssim B\right)$. It is trivial to check that this is a preorder on $M_{2}$ that extends $\lesssim$ (v., proof of Theorem 1 in Alcantud [2]). It verifies Axioms A, P, and $S$ (v., Remark 2). But it that does not verify Axiom $C$, since $M_{2} \lesssim M_{1}$ but $\operatorname{rank}\left(M_{1}\right)<\operatorname{rank}\left(M_{2}\right)$.

\subsection{Main result.}

Now we are ready to solve the problem we have posed ourselves.

Theorem 2 Let $\left\{A_{i}\right\}_{i=1, \ldots, m}$ and $\left\{B_{i}\right\}_{i=1, \ldots, m}$ be two ordered lists of matrices with $n$ columns. The following statements are equivalent:

a) There exists $R$ complete preorder on $M_{n}$ that satisfies Attribute-independence, Anonymity, Preference for average allocations, Compatibility with the rank and Scale invariance, and declares $B_{i} P_{R} A_{i}$ for each $i=1, \ldots, m$.

b) $\lesssim^{A}$ associated with $\left(A_{1}, \ldots, A_{m}, B_{1}, \ldots, B_{m}\right)$ and $\lesssim i s$ acyclic, and $\operatorname{rank}\left(B_{i}\right) \leqslant$ $\operatorname{rank}\left(A_{i}\right)$ for each $i=1, \ldots, m$.

\section{Proof:}

In order to check $a) \Rightarrow b$ ) we prove $\lesssim \subseteq R$ and then we use the fact that implication $a) \Rightarrow b$ ) in Theorem 1 only required inclusion instead of extension. 
Select $A, B$ matrices with $n$ columns, and suppose that there are $k>0, Q$ rowstochastic matrix and $T$ permutation matrix with $A=k Q B T$. By Scale invariance and Attribute-independence, $(k B T) I_{R} B T I_{R} B$. Now the fact $A=Q(k B T)$ plus Preference for average allocations entail $A R(k B T)$. Because $R$ is a complete preorder and $A R(k B T) I_{R} B$ we conclude $A R B$. This completes the argument. Furthermore, $\operatorname{rank}\left(B_{i}\right)>\operatorname{rank}\left(A_{i}\right)$ and $B_{i} R A_{i}$ are incompatible because $R$ satisfies Compatibility with the rank.

Let us now prove $b) \Rightarrow a$ ). By Theorem 1 we can assure that there is a complete preorder $\widetilde{R}$ on $M_{n}$ that extends $\lesssim$ and satisfies $B_{i} \widetilde{P} A_{i}$ for each $i=1, \ldots, m$.

Now let $R$ be the lexicographic relation defined by: $A R B$ if and only if either $\operatorname{rank}(A)<\operatorname{rank}(B)$ or $(\operatorname{rank}(A)=\operatorname{rank}(B)$ and $A \widetilde{R} B)$. It is a complete preorder that includes $\lesssim$ and thus it agrees with Attribute-independence, Anonymity, Preference for average allocations, and Scale invariance. Obviously it satisfies Compatibility with the rank. In order to check that $R$ fulfils the initial constraints, we only need to observe that $R$ 's asymmetric part $P$ is given by: $A P B$ if and only if either $\operatorname{rank}(A)<\operatorname{rank}(B)$ or $(\operatorname{rank}(A)=\operatorname{rank}(B)$ and $A \widetilde{P} B)$. Coupled with $B_{i} \widetilde{P} A_{i}$ and $\operatorname{rank}\left(B_{i}\right) \leqslant \operatorname{rank}\left(A_{i}\right)$ for each $i=1, \ldots, m$, this entails $B_{i} P A_{i}$ for each $i=1, \ldots, m$.

\section{Conclusions and final remarks.}

A conceptual problem that appears in different contexts of inequality analysis consists of measuring the degree of inequality in the data. This problem can be addressed by means of numerical indices or other methods. The lack of agreement on a procedure for measuring multidimensional inequality poses difficulties for checking if a given prescription is in accordance with theoretical predictions or not. Here we circunvent that handicap by appealing to the approach by Savaglio [19]. Our approach is novel in that we are interested in testing if complete methods of comparison that perform well exist, which do not contradict a finite list of ex-ante prescriptions. Formally, we state our problem in terms of the compatibility of a set of $m$ primitive inequality assessments with a comparison of multidimensional inequality by a model that fits into the mainstream of the field. By virtue of Theorem 2, a solution can be implemented in two parts. Firstly we must compute $2 m$ ranks of matrices with $n$ columns, and check if certain inequalities hold true. Suppose that this is the case (otherwise the initial constraints are incompatible with a measure of multidimensional inequality in the model). Then we must check if a binary relation that is trivially defined from raw data has cycles, or equivalently, if its transitive closure is irreflexive. For the case of few restrictions this can be performed by simple computations. But when the number of conditions increases we need to be assisted by algorithms. The literature abounds with proposals for computing the transitive closure of a binary relation efficiently. Varian [27, Appendix II] popularized the Floyd-Warshall algorithm (cf., [10], [28]) in the economic literature. The computer science literature offers further algorithms that are computationally more efficient (cf., e.g., Agrawal and Jagadish [1], Ioannidis and Ramakrishnan 
[12], Schmitz [22], or Schnorr [21]).

\section{Acknowledgement.}

The research reported here was supported by the Spanish Ministerio de Economía y Competitividad under Project ECO2012-31933. The author is indebted to the Editor and two anonymous referees for useful comments and suggestions.

\section{References}

[1] R. Agrawal, H.V. Jagadish, Efficient transitive closure algorithms, Proceedings of the 14th VLDB Conference (1988), 382-394.

[2] J.C.R. Alcantud, Conditional ordering extensions, Economic Theory 39 (2009), 495-503.

[3] J.C.R. Alcantud, R. de Andrés Calle, J. M. Cascón, A unifying model to measure consensus solutions in a society, Mathematical and Computer Modelling 57 (2013), 18761883.

[4] C.D. Aliprantis, K.C. Border, Infinite-dimensional analysis, 2nd edition, Springer-Verlag, 1999.

[5] A. Andrikopoulos, Szpilrajn-type theorems in economics, MPRA paper No.14345 (2009).

[6] A.B. Atkinson, On the measurement of inequality, Journal of Economic Theory 2 (1970), 244-263.

[7] A.B. Atkinson, F. Bourguignon, The comparison of multi-dimensioned distributions of economic status, Review of Economic Studies 49 (1982), 183-201.

[8] B. Chandra, P.P. Varghese, Moving towards efficient decision tree construction, Information Sciences 179 (2009), 1059-1069.

[9] T. Demuynck, Common ordering extensions. Working Paper 09/593, Faculty of Economics and Business Administration, Ghent University (2009).

[10] R.W. Floyd, A modification of Warshall's algorithm for the transitive closure of binary relations, Communications of the Association for Computing Machinery 18 (1975), 218220 .

[11] R.A. Horn, C.R. Johnson, Matrix Analysis, Cambridge University Press, 1985.

[12] Y. Ioannidis, R. Ramakrishnan, Efficient transitive closure algorithms. In: Proceedings of the 14th VLDB Conference (1988), 382-394.

[13] I. Koprinska, J. Poon, J. Clark, J. Chan, Learning to classify e-mail, Information Sciences 177 (2007), 2167-2187.

[14] Y.-Y. Lur, Y.-K. Wu, S.-M. Guu, Convergence of powers for a fuzzy matrix with convex combination of maxmin and max-arithmetic mean operations, Information Sciences 179 (2009), 938-944. 
[15] E. Maasoumi, The measurement and decomposition of multi-dimensional inequality, Econometrica 54 (1986), 991-997

[16] E. Maasoumi, A compendium to information theory in Economics and Econometrics, Econometric Review, 12 (2) (1993), 137-181.

[17] E. Maasoumi, Multidimensioned approaches to welfare analysis. In: J. Silber (Ed.), Handbook of income inequality measurement, Kluwer, 1999, Boston, pp. 437-477.

[18] C. Ok, S. Lee, P. Mitra, S. Kumara, Distributed routing in wireless sensor networks using energy welfare metric, Information Sciences 180 (2010), 1656-1670.

[19] E. Savaglio, Multidimensional inequality with variable population size, Economic Theory 28 (2006), 85-94.

[20] E. Savaglio, Multidimensional inequality: a survey. In: F. Farina and E. Savaglio (Eds.), Inequality and Economic Integration, Routledge/Taylor and Francis Group, 2006.

[21] C.P. Schnorr, An algorithm for transitive closure with linear expected time, SIAM Journal on Computing 7 (1978), 124-133.

[22] L. Schmitz, An improved transitive closure algorithm, Computing 30 (1983), 359-371.

[23] E. Szpilrajn, Sur l'extension de l'ordre partiel, Fundamenta Mathematicae 16 (1930), 386-389.

[24] H. Theil, Economics and Information Theory, North-Holland Amsterdam, 1967.

[25] K.Y. Tsui, Multidimensional generalizations of the relative and absolute inequality indices: The Atkinson-Kolm-Sen approach, Journal of Economic Theory 67 (1995), 251265

[26] U.S. Census Bureau, Measures of Household Income Inequality: 1967 to 2001. http://www.census.gov/hhes/www/income/histinc/ie6.html

[27] H. R. Varian, The nonparametric approach to demand analysis, Econometrica 50 (1982), 945-974.

[28] S. Warshall, A theorem on Boolean matrices, Journal of the American Association of Computing Machinery 9(1) (1962), 11-12.

[29] J. Weymark, The normative approach to the measurement of multidimensional inequality. In: F. Farina and E. Savaglio (Eds.), Inequality and Economic Integration, 2006, Routledge/Taylor and Francis Group. 